\title{
Parametrized inequality of Hermite-Hadamard type for functions whose third derivative absolute values are quasi-convex
}

\author{
Shan-He Wu ${ }^{1}$, Banyat Sroysang ${ }^{2}$, Jin-Shan Xie ${ }^{1}$ and Yu-Ming Chu ${ }^{3^{*}}$
}

\author{
${ }^{*}$ Correspondence: \\ chuyuming@aliyun.com; \\ chuyuming2005@126.com \\ 3 School of Mathematics \\ and Computation Science, \\ Hunan City University, \\ Yiyang 413000, Hunan, \\ People's Republic of China \\ Full list of author information \\ is available at the end of the \\ article
}

\begin{abstract}
In this paper we present some inequalities of Hermite-Hadamard type for functions whose third derivative absolute values are quasi-convex. Moreover, an application to special means of real numbers is also considered.
\end{abstract}

Keywords: Hermite-Hadamard type inequality, Parameter, Quasi-convex, Special means

Mathematics Subject Classification: 26D10, 26A51, 26E60

\section{Background}

A real-valued function $f$ defined on an interval $I \subseteq \mathbb{R}$ is said to be convex on $I$, if

$$
f(\lambda x+(1-\lambda) y) \leq \lambda f(x)+(1-\lambda) f(y)
$$

for all $x, y \in I$ and $\lambda \in[0,1]$.

If $f$ is convex on $I$, then we have the Hermite-Hadamard inequality (see Mitrinović et al. 1993)

$$
f\left(\frac{a+b}{2}\right) \leq \frac{1}{b-a} \int_{a}^{b} f(x) d x \leq \frac{f(a)+f(b)}{2}
$$

for all $a, b \in I$.

A function $f: I \subseteq \mathbb{R} \rightarrow \mathbb{R}$ is said to be quasi-convex on $I$, if

$$
f(\lambda x+(1-\lambda) y) \leq \max \{f(x), f(y)\}
$$

for all $x, y \in I$ and $\lambda \in[0,1]$.

Clearly, any convex function is a quasi-convex function. Furthermore, there exist quasi-convex functions which are not convex.

In 2007, Ion (2007) presented an inequality of Hermite-Hadamard type for functions whose derivatives in absolute values are quasi-convex functions, as follows:

\section{包 Springer}


Theorem 1.1 Let $f: I \subset \mathbb{R} \rightarrow \mathbb{R}$ be a differentiable mapping on $I^{o}, a, b \in I$ with $a<b$. If $\left|f^{\prime}\right|$ is quasi-convex on $[a, b]$, then the following inequality holds:

$$
\left|\frac{f(a)+f(b)}{2}-\frac{1}{b-a} \int_{a}^{b} f(x) d x\right| \leq \frac{b-a}{4} \max \left\{\left|f^{\prime}(a)\right|,\left|f^{\prime}(b)\right|\right\} .
$$

In 2010, Alomari et al. (2010a) established an analogous version of inequality (1), which is asserted by Theorem 1.2 below:

Theorem 1.2 Let $f: I \subset \mathbb{R} \rightarrow \mathbb{R}$ be twice differentiable mapping on $I^{o}, a, b \in I$ with $a<b$ and $f^{\prime \prime}$ is integrable on $[a, b]$. If $\left|f^{\prime \prime}\right|$ is quasi-convex on $[a, b]$, then the following inequality holds:

$$
\left|\frac{f(a)+f(b)}{2}-\frac{1}{b-a} \int_{a}^{b} f(x) d x\right| \leq \frac{(b-a)^{2}}{12} \max \left\{\left|f^{\prime \prime}(a)\right|,\left|f^{\prime \prime}(b)\right|\right\} .
$$

Recently, Guo et al. (2015) investigated Hermite-Hadamard type inequalities for geometrically quasi-convex functions. Xi and Qi $(2014,2015)$ and Xi et al. $(2012,2014)$ showed some new Hermite-Hadamard type inequalities for s-convex functions. For more results relating to refinements, counterparts, generalizations of Hadamard type inequalities, we refer interested readers to Alomari et al. (2010b), Chen (2015), Niculescu and Persson (2006), Pečarić et al. (1992), Sroysang (2014), Sroysang (2013) and $\mathrm{Wu}(2009)$.

The main purpose of this paper is to present a parametrized inequality of HermiteHadamard type for functions whose third derivative absolute values are quasi-convex. As applications, some new inequalities for special means of real numbers are established.

\section{Lemmas}

In order to prove our main results, we need the following lemmas.

Lemma 2.1 Let $\in \in \mathbb{R}$ and let $f: I \subset \mathbb{R} \rightarrow \mathbb{R}$ be three times differentiable on $I^{\circ}$ and $a, b \in I$ with $a<b$. Assume that $f^{\prime \prime \prime}$ is integrable on $[a, b]$. Then

$$
\begin{gathered}
\frac{(4-\epsilon) f(a)+(2+\epsilon) f(b)}{6}-\frac{1}{b-a} \int_{a}^{b} f(x) d x-\frac{b-a}{12}\left(\epsilon f^{\prime}(b)-(2-\epsilon) f^{\prime}(a)\right) \\
=\frac{(b-a)^{3}}{12} \int_{0}^{1} \lambda(1-\lambda)(2 \lambda-\epsilon) f^{\prime \prime \prime}(\lambda a+(1-\lambda) b) d \lambda .
\end{gathered}
$$


Proof Integrating by parts, we have

$$
\begin{aligned}
& \int_{0}^{1} \lambda(1-\lambda)(2 \lambda-\epsilon) f^{\prime \prime \prime}(\lambda a+(1-\lambda) b) d \lambda \\
& =\frac{1}{a-b} \int_{\lambda=0}^{\lambda=1} \lambda(1-\lambda)(2 \lambda-\epsilon) d f^{\prime \prime}(\lambda a+(1-\lambda) b) \\
& =\frac{1}{a-b}\left[\lambda(1-\lambda)(2 \lambda-\epsilon) f^{\prime \prime}(\lambda a+(1-\lambda) b)\right]_{\lambda=0}^{\lambda=1} \\
& -\frac{1}{a-b} \int_{\lambda=0}^{\lambda=1} f^{\prime \prime}(\lambda a+(1-\lambda) b) d(\lambda(1-\lambda)(2 \lambda-\epsilon)) \\
& =-\frac{1}{a-b} \int_{\lambda=0}^{\lambda=1} f^{\prime \prime}(\lambda a+(1-\lambda) b) d(\lambda(1-\lambda)(2 \lambda-\epsilon)) \\
& =\frac{1}{b-a} \int_{\lambda=0}^{\lambda=1}\left(-6 \lambda^{2}+2(2+\epsilon) \lambda-\epsilon\right) f^{\prime \prime}(\lambda a+(1-\lambda) b) d \lambda \\
& =\frac{1}{(b-a)^{2}} \int_{\lambda=0}^{\lambda=1}\left(6 \lambda^{2}-2(2+\epsilon) \lambda+\epsilon\right) d f^{\prime}(\lambda a+(1-\lambda) b) \\
& =\frac{1}{(b-a)^{2}}\left[\left(6 \lambda^{2}-2(2+\epsilon) \lambda+\epsilon\right) f^{\prime}(\lambda a+(1-\lambda) b)\right]_{\lambda=0}^{\lambda=1} \\
& -\frac{1}{(b-a)^{2}} \int_{\lambda=0}^{\lambda=1} f^{\prime}(\lambda a+(1-\lambda) b) d\left(6 \lambda^{2}-2(2+\epsilon) \lambda+\epsilon\right) \\
& =\frac{-1}{(b-a)^{2}}\left(\epsilon f^{\prime}(b)-(2-\epsilon) f^{\prime}(a)\right) \\
& -\frac{1}{(b-a)^{2}} \int_{\lambda=0}^{\lambda=1}(12 \lambda-2(2+\epsilon)) f^{\prime}(\lambda a+(1-\lambda) b) d \lambda \\
& =\frac{-1}{(b-a)^{2}}\left(\epsilon f^{\prime}(b)-(2-\epsilon) f^{\prime}(a)\right) \\
& +\frac{2}{(b-a)^{3}} \int_{\lambda=0}^{\lambda=1}(6 \lambda-2-\epsilon) d f(\lambda a+(1-\lambda) b) \\
& =\frac{-1}{(b-a)^{2}}\left(\epsilon f^{\prime}(b)-(2-\epsilon) f^{\prime}(a)\right) \\
& +\frac{2}{(b-a)^{3}}[(6 \lambda-2-\epsilon) f(\lambda a+(1-\lambda) b)]_{\lambda=0}^{\lambda=1} \\
& -\frac{2}{(b-a)^{3}} \int_{\lambda=0}^{\lambda=1} f(\lambda a+(1-\lambda) b) d(6 \lambda-2-\epsilon) \\
& =\frac{-1}{(b-a)^{2}}\left(\epsilon f^{\prime}(b)-(2-\epsilon) f^{\prime}(a)\right) \\
& +\frac{2}{(b-a)^{3}}((4-\epsilon) f(a)+(2+\epsilon) f(b)) \\
& -\frac{12}{(b-a)^{3}} \int_{\lambda=0}^{\lambda=1} f(\lambda a+(1-\lambda) b) d \lambda .
\end{aligned}
$$


Changing variable $x=\lambda a+(1-\lambda) b$, it follows that

$$
\begin{aligned}
\int_{0}^{1} & \lambda(1-\lambda)(2 \lambda-\epsilon) f^{\prime \prime \prime}(\lambda a+(1-\lambda) b) d \lambda \\
= & \frac{-1}{(b-a)^{2}}\left(\epsilon f^{\prime}(b)-(2-\epsilon) f^{\prime}(a)\right) \\
& +\frac{2}{(b-a)^{3}}((4-\epsilon) f(a)+(2+\epsilon) f(b)) \\
& -\frac{12}{(b-a)^{4}} \int_{x=a}^{x=b} f(x) d x .
\end{aligned}
$$

Thus,

$$
\begin{aligned}
& \frac{(b-a)^{3}}{12} \int_{0}^{1} \lambda(1-\lambda)(2 \lambda-\epsilon) f^{\prime \prime \prime}(\lambda a+(1-\lambda) b) d \lambda \\
& \quad=\frac{(4-\epsilon) f(a)+(2+\epsilon) f(b)}{6}-\frac{1}{b-a} \int_{a}^{b} f(x) d x-\frac{b-a}{12}\left(\epsilon f^{\prime}(b)-(2-\epsilon) f^{\prime}(a)\right) .
\end{aligned}
$$

The proof of Lemma 2.1 is completed.

Lemma 2.2 Let $\epsilon$ be a real number. Then

$$
\int_{0}^{1} \lambda(1-\lambda)|2 \lambda-\epsilon| d \lambda= \begin{cases}\frac{\epsilon-1}{6} & \text { if } \epsilon \geq 2 \\ \frac{4 \epsilon^{3}-\epsilon^{4}}{48}+\frac{1-\epsilon}{6} & \text { if } 0<\epsilon<2 \\ \frac{1-\epsilon}{6} & \text { if } \epsilon \leq 0 .\end{cases}
$$

Proof We distinguish three cases

Case 1 If $\epsilon \geq 2$, then

$$
\int_{0}^{1} \lambda(1-\lambda)|2 \lambda-\epsilon| d \lambda=\int_{0}^{1} \lambda(1-\lambda)(\epsilon-2 \lambda) d \lambda=\frac{\epsilon-1}{6} .
$$

Case 2 If $0<\epsilon<2$, then

$$
\begin{aligned}
\int_{0}^{1} \lambda(1-\lambda)|2 \lambda-\epsilon| d \lambda & =\int_{0}^{\epsilon / 2} \lambda(1-\lambda)(\epsilon-2 \lambda) d \lambda+\int_{\epsilon / 2}^{1} \lambda(1-\lambda)(2 \lambda-\epsilon) d \lambda \\
& =\frac{4 \epsilon^{3}-\epsilon^{4}}{48}+\frac{1-\epsilon}{6} .
\end{aligned}
$$

Case 3 If $\epsilon \leq 0$, then

$$
\int_{0}^{1} \lambda(1-\lambda)|2 \lambda-\epsilon| d \lambda=\int_{0}^{1} \lambda(1-\lambda)(2 \lambda-\epsilon) d \lambda=\frac{1-\epsilon}{6} .
$$

This completes the proof of Lemma 2.2. 
Main results

Our main results are stated in the following theorems.

Theorem 3.1 Let $q \geq 1$ and $\in \in \mathbb{R}$, and let $f: I \subset \mathbb{R} \rightarrow \mathbb{R}$ be three times differentiable on $I^{\circ}$ and $a, b \in I$ with $a<b$. Assume that $f^{\prime \prime \prime}$ is integrable on $[a, b]$, and $\left|f^{\prime \prime \prime}\right|^{q}$ is quasiconvex on $[a, b]$. Then

$$
\begin{aligned}
& \left|\frac{(4-\epsilon) f(a)+(2+\epsilon) f(b)}{6}-\frac{1}{b-a} \int_{a}^{b} f(x) d x-\frac{b-a}{12}\left(\epsilon f^{\prime}(b)-(2-\epsilon) f^{\prime}(a)\right)\right| \\
& \quad \leq \begin{cases}\frac{(b-a)^{3}}{12}\left(\frac{\epsilon-1}{6}\right)\left(\max \left\{\left|f^{\prime \prime \prime}(a)\right|^{q},\left|f^{\prime \prime \prime}(b)\right|^{q}\right\}\right)^{1 / q} & \text { if } \epsilon \geq 2 \\
\frac{(b-a)^{3}}{12}\left(\frac{4 \epsilon^{3}-\epsilon^{4}}{48}+\frac{1-\epsilon}{6}\right)\left(\max \left\{\left|f^{\prime \prime \prime}(a)\right|^{q},\left|f^{\prime \prime \prime}(b)\right|^{q}\right\}\right)^{1 / q} & \text { if } 0<\epsilon<2 \\
\frac{(b-a)^{3}}{12}\left(\frac{1-\epsilon}{6}\right)\left(\max \left\{\left|f^{\prime \prime \prime}(a)\right|^{q},\left|f^{\prime \prime \prime}(b)\right|^{q}\right\}\right)^{1 / q} & \text { if } \epsilon \leq 0 .\end{cases}
\end{aligned}
$$

Proof Using Lemma 2.1 and Hölder's inequality gives

$$
\begin{aligned}
& \mid \frac{(4-\epsilon) f(a)+(2+\epsilon) f(b)}{6}-\frac{1}{b-a} \int_{a}^{b} f(x) d x-\frac{b-a}{12}\left(\epsilon f^{\prime}(b)-(2-\epsilon) f^{\prime}(a)\right) \mid \\
& \leq \frac{(b-a)^{3}}{12} \int_{0}^{1} \lambda(1-\lambda)|2 \lambda-\epsilon|\left|f^{\prime \prime \prime}(\lambda a+(1-\lambda) b)\right| d \lambda \\
&= \frac{(b-a)^{3}}{12} \int_{0}^{1}(\lambda(1-\lambda)|2 \lambda-\epsilon|)^{1-1 / q} \\
& \times\left(\lambda(1-\lambda)|2 \lambda-\epsilon|\left|f^{\prime \prime \prime}(\lambda a+(1-\lambda) b)\right|^{q}\right)^{1 / q} d \lambda \\
& \leq \frac{(b-a)^{3}}{12}\left(\int_{0}^{1} \lambda(1-\lambda)|2 \lambda-\epsilon| d \lambda\right)^{1-1 / q} \\
& \quad \times\left(\int_{0}^{1} \lambda(1-\lambda)|2 \lambda-\epsilon|\left|f^{\prime \prime \prime}(\lambda a+(1-\lambda) b)\right|^{q} d \lambda\right)^{1 / q} .
\end{aligned}
$$

By the quasi-convexity of $\left|f^{\prime \prime \prime}\right|^{q}$, we obtain

$$
\begin{aligned}
& \left|\frac{(4-\epsilon) f(a)+(2+\epsilon) f(b)}{6}-\frac{1}{b-a} \int_{a}^{b} f(x) d x-\frac{b-a}{12}\left(\epsilon f^{\prime}(b)-(2-\epsilon) f^{\prime}(a)\right)\right| \\
& \leq \frac{(b-a)^{3}}{12}\left(\int_{0}^{1} \lambda(1-\lambda)|2 \lambda-\epsilon| d \lambda\right)^{1-1 / q} \\
& \quad \times\left(\int_{0}^{1} \lambda(1-\lambda)|2 \lambda-\epsilon| \max \left\{\left|f^{\prime \prime \prime}(a)\right|^{q},\left|f^{\prime \prime \prime}(b)\right|^{q}\right\} d \lambda\right)^{1 / q} \\
& =\frac{(b-a)^{3}}{12}\left(\int_{0}^{1} \lambda(1-\lambda)|2 \lambda-\epsilon| d \lambda\right)\left(\max \left\{\left|f^{\prime \prime \prime}(a)\right|^{q},\left|f^{\prime \prime \prime}(b)\right|^{q}\right\}\right)^{1 / q} .
\end{aligned}
$$


Utilizing Lemma 2.2 leads to the desired inequality in Theorem 3.1.

Remark 3.2 It is worth noticing that if we use a substitution $a \rightarrow b, b \rightarrow a$ and $\epsilon \rightarrow 2-\epsilon$ in Theorem 3.1, we have the following further generalization of Theorem 3.1.

Theorem 3.3 Let $q \geq 1$ and $\in \in \mathbb{R}$, and let $f: I \subset \mathbb{R} \rightarrow \mathbb{R}$ be three times differentiable on $I^{\circ}, a, b \in I$ with $a \neq b$. Assume that $f^{\prime \prime \prime}$ is integrable on $[a, b]$, and $\left.\left.\right|^{\prime \prime \prime}\right|^{q}$ is quasi-convex on the closed interval formed by the points $a$ and $b$. Then

$$
\begin{aligned}
& \left|\frac{(4-\epsilon) f(a)+(2+\epsilon) f(b)}{6}-\frac{1}{b-a} \int_{a}^{b} f(x) d x-\frac{b-a}{12}\left(\epsilon f^{\prime}(b)-(2-\epsilon) f^{\prime}(a)\right)\right| \\
& \quad \leq \begin{cases}\frac{|b-a|^{3}}{12}\left(\frac{\epsilon-1}{6}\right)\left(\max \left\{\left|f^{\prime \prime \prime}(a)\right|^{q},\left|f^{\prime \prime \prime}(b)\right|^{q}\right\}\right)^{1 / q} & \text { if } \epsilon \geq 2 \\
\frac{|b-a|^{3}}{12}\left(\frac{4 \epsilon^{3}-\epsilon^{4}}{48}+\frac{1-\epsilon}{6}\right)\left(\max \left\{\left|f^{\prime \prime \prime}(a)\right|^{q},\left|f^{\prime \prime \prime}(b)\right|^{q}\right\}\right)^{1 / q} & \text { if } 0<\epsilon<2 \\
\frac{|b-a|^{3}}{12}\left(\frac{1-\epsilon}{6}\right)\left(\max \left\{\left|f^{\prime \prime \prime}(a)\right|^{q},\left|f^{\prime \prime \prime}(b)\right|^{q}\right\}\right)^{1 / q} & \text { if } \epsilon \leq 0 .\end{cases}
\end{aligned}
$$

As a direct consequence, choosing $\epsilon=1$ in Theorem 3.3, we get the following inequality:

Corollary 3.4 Let $q \geq 1$ and $\epsilon \in \mathbb{R}$, and let $f: I \subset \mathbb{R} \rightarrow \mathbb{R}$ be three times differentiable on $I^{\circ}, a, b \in I$ with $a \neq b$. Assume that $f^{\prime \prime \prime}$ is integrable on $[a, b]$, and $\left|f^{\prime \prime \prime}\right|^{q}$ is quasi-convex on the closed interval formed by the points $a$ and $b$. Then

$$
\begin{aligned}
& \left|\frac{f(a)+f(b)}{2}-\frac{1}{b-a} \int_{a}^{b} f(x) d x-\frac{b-a}{12}\left(f^{\prime}(b)-f^{\prime}(a)\right)\right| \\
& \leq \frac{|b-a|^{3}}{192}\left(\max \left\{\left|f^{\prime \prime \prime}(a)\right|^{q},\left|f^{\prime \prime \prime}(b)\right|^{q}\right\}\right)^{1 / q} .
\end{aligned}
$$

In addition, if we utilize Theorem 3.1 with a substitution of $\epsilon=0,0.5,3,-2,-3,-5$, respectively, then we obtain the following results:

Corollary 3.5 Let $q \geq 1$ and $\in \in \mathbb{R}$, and let $f: I \subset \mathbb{R} \rightarrow \mathbb{R}$ be three times differentiable on $I^{\circ}, a, b \in I$ with $a \neq b$. Assume that $f^{\prime \prime \prime}$ is integrable on $[a, b]$, and $\left|f^{\prime \prime \prime}\right|^{q}$ is quasi-convex on the closed interval formed by the points $a$ and $b$. Then

$$
\begin{aligned}
& \left|\frac{2 f(a)+f(b)}{3}-\frac{1}{b-a} \int_{a}^{b} f(x) d x+\frac{b-a}{6} f^{\prime}(a)\right| \\
& \leq \frac{|b-a|^{3}}{72}\left(\max \left\{\left|f^{\prime \prime \prime}(a)\right|^{q},\left|f^{\prime \prime \prime}(b)\right|^{q}\right\}\right)^{1 / q}, \\
& \left|\frac{7 f(a)+5 f(b)}{12}-\frac{1}{b-a} \int_{a}^{b} f(x) d x-\frac{b-a}{24}\left(f^{\prime}(b)-3 f^{\prime}(a)\right)\right| \\
& \quad \leq \frac{71|b-a|^{3}}{9216}\left(\max \left\{\left|f^{\prime \prime \prime}(a)\right|^{q},\left|f^{\prime \prime \prime}(b)\right|^{q}\right\}\right)^{1 / q},
\end{aligned}
$$




$$
\begin{aligned}
& \left|\frac{f(a)+5 f(b)}{6}-\frac{1}{b-a} \int_{a}^{b} f(x) d x-\frac{b-a}{12}\left(3 f^{\prime}(b)+f^{\prime}(a)\right)\right| \\
& \leq \frac{|b-a|^{3}}{36}\left(\max \left\{\left|f^{\prime \prime \prime}(a)\right|^{q},\left|f^{\prime \prime \prime}(b)\right|^{q}\right\}\right)^{1 / q}, \\
& \left|f(a)-\frac{1}{b-a} \int_{a}^{b} f(x) d x+\frac{b-a}{6}\left(f^{\prime}(b)+2 f^{\prime}(a)\right)\right| \\
& \leq \frac{|b-a|^{3}}{24}\left(\max \left\{\left|f^{\prime \prime \prime}(a)\right|^{q},\left|f^{\prime \prime \prime}(b)\right|^{q}\right\}\right)^{1 / q}, \\
& \left|\frac{7 f(a)-f(b)}{6}-\frac{1}{b-a} \int_{a}^{b} f(x) d x+\frac{b-a}{12}\left(3 f^{\prime}(b)+5 f^{\prime}(a)\right)\right| \\
& \leq \frac{|b-a|^{3}}{18}\left(\max \left\{\left|f^{\prime \prime \prime}(a)\right|^{q},\left|f^{\prime \prime \prime}(b)\right|^{q}\right\}\right)^{1 / q}, \\
& \left|\frac{3 f(a)-f(b)}{2}-\frac{1}{b-a} \int_{a}^{b} f(x) d x+\frac{b-a}{12}\left(5 f^{\prime}(b)+7 f^{\prime}(a)\right)\right| \\
& \leq \frac{|b-a|^{3}}{12}\left(\max \left\{\left|f^{\prime \prime \prime}(a)\right|^{q},\left|f^{\prime \prime \prime}(b)\right|^{q}\right\}\right)^{1 / q} \text {. }
\end{aligned}
$$

\section{Applications to special means}

We now consider the applications of our results to the special means of real numbers.

The weighted arithmetic mean of real numbers $\{a, b\}$ with weight $\left\{w_{a}, w_{b}\right\}$ is defined by

$$
A\left(a, b ; w_{a}, w_{b}\right)=\frac{w_{a} a+w_{b} b}{w_{a}+w_{b}},
$$

where $a, b, w_{a}, w_{b} \in \mathbb{R}$ with $w_{a}+w_{b} \neq 0$.

In particularly

$$
A(a, b ; 1,1)=A(a, b)=\frac{a+b}{2},
$$

which is called the arithmetic means.

The generalized logarithmic mean of real numbers $\{a, b\}$ is defined by

$$
L_{n}(a, b)=\left[\frac{b^{n+1}-a^{n+1}}{(n+1)(b-a)}\right]^{\frac{1}{n}},
$$

where $a, b, \in \mathbb{R}, n \in \mathbb{Z}$ with $n \neq 0,-1, a \neq b$. 
Proposition 4.1 Let $a, b, \epsilon \in \mathbb{R}, a \neq b, \epsilon \neq 1$ and $n \in \mathbb{N}, n \geq 3$. Then, we have

$$
\begin{aligned}
& \left|A\left(a^{n}, b^{n} ; 4-\epsilon, 2+\epsilon\right)-\frac{(b-a)(\epsilon-1) n}{6} A\left(a^{n-1}, b^{n-1} ; \epsilon-2, \epsilon\right)-L_{n}^{n}(a, b)\right| \\
& \leq \begin{cases}\frac{|b-a|^{3}}{72}(\epsilon-1) n(n-1)(n-2)\left(\max \left\{|a|^{n-3},|b|^{n-3}\right\}\right) & \text { if } \epsilon \geq 2 \\
\frac{|b-a|^{3}}{576}\left(8-8 \epsilon+4 \epsilon^{3}-\epsilon^{4}\right) n(n-1)(n-2)\left(\max \left\{|a|^{n-3},|b|^{n-3}\right\}\right) & \text { if } 0<\epsilon<2 \\
\frac{|b-a|^{3}}{72}(1-\epsilon) n(n-1)(n-2)\left(\max \left\{|a|^{n-3},|b|^{n-3}\right\}\right) & \text { if } \epsilon \leq 0 .\end{cases}
\end{aligned}
$$

Proof We consider the function $f(x)=x^{n}, x \in \mathbb{R}, n \geq 3$. It is easy to verify that the function $f^{\prime \prime \prime}(x)=n(n-1)(n-2) x^{n-3}$ is quasi-convex on $(-\infty,+\infty)$ (see Alomari et al. 2010b). The assertion follows from Theorem 3.3 with $q=1$.

Remark 4.2 In a similar way as the proof of the Proposition 4.1, one can easily deduce from Corollary 3.4 the following inequality.

Proposition 4.3 Let $a, b \in \mathbb{R}, a \neq b$ and $n \in \mathbb{N}, n \geq 3$. Then, we have

$$
\begin{aligned}
& \left|A\left(a^{n}, b^{n}\right)-\frac{(b-a)^{2}}{12} n(n-1) L_{n-2}^{n-2}(a, b)-L_{n}^{n}(a, b)\right| \\
& \quad \leq \frac{|b-a|^{3}}{192} n(n-1)(n-2)\left(\max \left\{|a|^{n-3},|b|^{n-3}\right\}\right) .
\end{aligned}
$$

\section{Conclusions}

This paper provides some new results related to the Hermite-Hadamard type inequalities. Firstly, we present a parametrized inequality of Hermite-Hadamard type for functions whose third derivative absolute values are quasi-convex, the main results are given in Theorems 3.1 and 3.3. As special cases, by assigning special value to the parameter, one can obtain several new and previously known results for Hermite-Hadamard type inequality (Corollaries 3.4 and 3.5). Secondly, as applications of the obtained results, we establish two new inequalities involving special means of real numbers by using the parametrized Hermite-Hadamard type inequality (see Propositions 4.1 and 4.3).

Authors' contributions

SW finished the proof and the writing work. BS and JX gave SW some advice on the proof and writing. YC gave SW lots of help in revising the paper. All authors read and approved the final manuscript.

\section{Author details}

${ }^{1}$ Department of Mathematics, Longyan University, Longyan 364012, Fujian, People's Republic of China. ${ }^{2}$ Department of Mathematics and Statistics, Faculty of Science and Technology, Thammasat University, Pathumthani 12121, Thailand. ${ }^{3}$ School of Mathematics and Computation Science, Hunan City University, Yiyang 413000, Hunan, People's Republic of China.

\section{Acknowledgements}

The authors would like to express their hearty thanks to anonymous referees for their valuable comments on this article. This research was supported by the Natural Science Foundation of Fujian province under Grant 2015J05010 and the Outstanding Young Incubation Programme and Key Project of Fujian Province Education Department under Grant JA14299.

\section{Competing interests}

The authors declare that they have no competing interests. 
Received: 8 October 2015 Accepted: 17 December 2015

Published online: 30 December 2015

\section{References}

Alomari M, Darus M, Dragomir SS (2010) New inequalities of Hermite-Hadamard type for functions whose second derivatives absolute values are quasi-convex. Tamkang J Math 41(4):353-359

Alomari M, Darus M, Kirmaci US (2010) Refinements of Hadamard-type inequalities for quasi-convex functions with applications to trapezoidal formula and to special means. Comput Math Appl 59:225-232

Chen FX (2015) Extensions of the Hermite-Hadamard inequality for harmonically convex functions via fractional integrals. Appl Math Comput 268(1):121-128

Guo XY, Qi F, Xi BY (2015) Some new Hermite-Hadamard type inequalities for geometrically quasi-convex functions on co-ordinates. J Nonlinear Sci Appl 8(5):740-749

Ion DA (2007) Some estimates on the Hermite-Hadamard inequality through quasi-convex functions, Annals of University of Craiova. Math Comp Sci Ser 34:82-87

Mitrinović DS, Pečarié JE, Fink AM (1993) Classical and New Inequalities in Analysis. Kluwer Academic Publishers, Dordrecht

Niculescu CP, Persson LE (2006) Convex functions and their applications: a contemporary approach. In: CMS Books in Mathematics, vol. 23. Springer, New York

Pečarić JE, Proschan F, Tong YL (1992) Convex functions, partial orderings, and statistical applications. Academic Press, New York

Sroysang B (2013) On the Hermite-Hadamard inequality and other integral inequalities involving several functions. J Funct Spaces Appl (Article ID 921828)

Sroysang B (2014) Generalizations on some Hermite-Hadamard type inequalities for differentiable convex functions with applications to weighted means. Sci World J (Article ID 717164)

Wu S (2009) On the weighted generalization of the Hermite-Hadamard inequality and its applications. Rocky Mt J Math 39(5):1741-1749

Xi BY, Bai RF, Qi F (2012) Hermite-Hadamard type inequalities for the $m$ - and $(\alpha ; m)$-geometrically convex functions. Aequ Math 84(3):261-269

Xi BY, Qi F (2014) Hermite-Hadamard type inequalities for geometrically r-convex functions. Studia Sci Math Hungarica 51(4):530-546

Xi BY, Qi F (2015) Inequalities of Hermite-Hadamard type for extended s-convex functions and applications to means. J Nonlinear Convex Anal 16(5):873-890

Xi BY, Wang SH, Qi F (2014) Some inequalities for $(h, m)$-convex functions. J Inequal Appl (Article ID 100)

\section{Submit your manuscript to a SpringerOpen ${ }^{\circ}$ journal and benefit from:}

- Convenient online submission

- Rigorous peer review

- Immediate publication on acceptance

- Open access: articles freely available online

- High visibility within the field

- Retaining the copyright to your article

Submit your next manuscript at $>$ springeropen.com 